\title{
An Aerothermoelastic Analysis Framework Enhanced by Model Order Reduction With Applications
}

\author{
Daning Huang*, Tomer Rokita ${ }^{\dagger}$ and Peretz P. Friedmann ${ }^{\ddagger}$ \\ Department of Aerospace Engineering
}

The University of Michigan, Ann Arbor, MI, 48109

\begin{abstract}
This study describes the reduction of the computer time requirements for an aerothermoelastic computational framework. The reduced order model (ROM) of the fluid solver is developed using kriging and proper orthogonal decomposition. The ROM accelerates the fluid solution by five orders of magnitude. In addition, a smart ordering scheme is proposed to accelerate the sample generation for the ROM. Using the extended computational framework, panel flutter problems are investigated with emphasis on flow orientation angle and boundary layer thickness. These parameters are found to have significant influence on the critical flutter dynamic pressure and amplitude of limit cycle oscillation.
\end{abstract}

\section{List of Symbols}

Latin symbols

\begin{tabular}{|c|c|}
\hline$a_{\infty}$ & Speed of sound \\
\hline$a, b$ & Panel dimensions \\
\hline $\mathbf{a}=\left[a_{1}, \cdots, a_{n_{S}}\right]$ & Modal coodinates of structure \\
\hline $\mathbf{b}=\left[b_{1}, \cdots, b_{n_{T}}\right]$ & Modal coodinates of wall temperature \\
\hline c & Flight condition \\
\hline $\mathrm{cov}$ & Covariance function \\
\hline$C_{H}=\dot{q} /\left[\rho U_{\infty} c_{p}\left(T-T_{w}\right)\right]$ & Stanton number \\
\hline$C_{p}=\left(p-p_{\infty}\right) / q_{\infty}$ & Pressure coefficient \\
\hline$c_{p}$ & Specific heat \\
\hline $\mathcal{D}$ & Sample data set \\
\hline$D=E h^{3} /\left[12\left(1-\nu^{2}\right)\right]$ & Bending stiffness of panel \\
\hline$d\left(u_{i}, u_{j}\right)$ & The distance between two fluid solutions \\
\hline$E$ & Young's modulus \\
\hline $\mathbf{f}=\left[f_{1}, \cdots, f_{q}\right]$ & Basis functions in global approximation in kriging model \\
\hline$h$ & Thickness of a skin panel \\
\hline$M$ & Mach number \\
\hline$N_{s}$ & Number of samples \\
\hline$n_{C}$ & Number of clusters in smart ordering scheme \\
\hline$n_{F}$ & Number of flight conditions \\
\hline$n_{S}, n_{T}$ & Number of modes for structure and wall temperature \\
\hline$p$ & Surface pressure \\
\hline $\mathbf{p}$ & Vector of pressure coefficients \\
\hline$q$ & Dynamic pressure \\
\hline$\dot{q}$ & Surface heat flux \\
\hline$\dot{\mathbf{q}}$ & Vector of Stanton numbers \\
\hline $\mathbf{R}$ & Correlation matrix \\
\hline$R$ & Correlation function \\
\hline$R e=\rho U_{\infty} a / \mu$ & Reynolds number \\
\hline$r$ & Number of POD modes \\
\hline $\mathbf{S}$ & Snapshot matrix \\
\hline
\end{tabular}

\footnotetext{
*Ph.D. Candidate, Student Member AIAA.

$\dagger$ Post Doctoral Research Fellow, Associate Member AIAA.

${ }^{\ddagger}$ François-Xavier Bagnoud Professor of Aerospace Engineering, Fellow AIAA.
} 


$\begin{array}{ll}T_{w} & \text { Wall temperature } \\ t & \text { Time } \\ \Delta t & \text { Time step } \\ \mathbf{U} & \text { Orthonormal matrix with columns as left singular vectors } \\ U & \text { Flow velocity } \\ u & \text { Fluid solution } \\ u_{0} & \text { Fluid field with undeformed panel and uniform wall temperature } \\ \mathbf{u} & \text { Left singular vectors } \\ \mathbf{V} & \text { Orthonormal matrix with columns as right singular vectors } \\ \mathbf{v} & \text { Right singular vectors } \\ w & \text { Displacement of the structure } \\ \mathbf{x} & \text { Sample point } \\ \mathbf{x}_{u}, \mathbf{x}_{l} & \text { Upper and Lower limits of input parameters } \\ \mathbf{y} & \text { Solution vector } \\ \mathbf{y} P O D-k r g & \text { The kriging interpolation function in POD-kriging model } \\ Z & \text { Local deviation in kriging model } \\ \Delta z & \text { The cell height of the fluid mesh }\end{array}$

Greek symbols

$\boldsymbol{\beta}$

$\beta$

$\delta$

$\epsilon$

$\Theta$

$\lambda=\rho U_{\infty}^{2} a^{3} / D$

$\lambda_{c r}$

$\mu$

$\mu_{s}=\rho a /\left(\rho_{m} h\right)$

$\rho$

$\rho_{m}$

$\Sigma$

$\sigma_{k r g}$

$\sigma$

$\psi_{S}, \psi_{T}$

$\Psi$

$\Psi_{p}$

$\boldsymbol{\Psi}_{q}$

\section{Acronyms}

Coefficients in global approximation

Flow orientation angle

Boundary layer thickness

Error

Diagonal coefficient matrix for correlation function

Nondimensional dynamic pressure

The critical value of $\lambda$

Air viscosity

Mass ratio

Air density

Structural density

Singular value matrix

Standard deviation in kriging model

Singular value

Mode for structure and wall temperature

POD matrix, whose columns are POD modes

POD matrix for pressure distribution

POD matrix for heat flux distribution

ADflow
CFD
FTSI
HYPATE
kNN
LCO
LnSp
MOR
MST
OLH
PETSc
POD
PT

Automatic Differentiation flow solver Computational Fluid Dynamics

Fluid-Thermal-Structural Interactions

HYPersonic AeroThermoElasticity simulation environment

The $k$-Nearest-Neighbour algorithm

Limit Cycle Oscillation

Linearized supersonic theory

Model Order Reduction

Minimum Spanning Tree

Optimal Latin Hypercube

Portable, Extensible Toolkit for Scientific computation

Proper Orthogonal Decomposition

Piston Theory 
RANS

ROM

SUmb

SVD

\section{Subscripts}

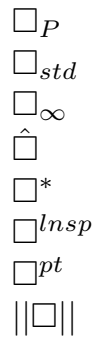

Reynolds-Averaged Navier-Stokes

Reduced Order Model

Stanford University multiblock

Singular Value Decomposition

Quantities expressed using POD modes

Quantities related to steady component of a dynamic solution

Quantities related to freestream

Quantities related to estimation

Quantities related to reference solution

Quantities related to linearized supersonic theory

Quantities related to piston theory

The $L^{2}$ norm of a vector

\section{Introduction}

Motivated by the interest in reusable launch vehicles for low-cost space exploration and rapid response to global military threats, hypersonic flight has been an intermittent area of research for decades [1]. Hypersonic vehicles operate at very high speeds and are exposed to extreme aerothermodynamic environments that involve extreme aerodynamic loading and heating. There are several unresolved technical challenges associated with hypersonic vehicles. An important issue in structural integrity is the simulation of the aerothermoelastic response over extended time periods corresponding to a vehicle trajectory. Sustained hypersonic flight causes severe aerodynamic heating and leads to degradation of material properties. The thermal stress introduced by the temperature gradients and geometrical constraints can dramatically affect structural stability and cause buckling, panel flutter, and control surface flutter. The analysis of performance, stability, and reliability of hypersonic vehicles requires accurate modeling of the complex hypersonic flow physics. A computational fluid dynamics (CFD) solver is needed to capture the essential properties of the flow physics. However, CFD-based aerothermoelastic dynamic simulation over the time scale of flight is intractable due to high computational cost. Therefore, a reduced order model (ROM) for the CFD solver is required to accelerate the performance of the fluid solver.

Popular model order reduction (MOR) techniques include surrogate-based methods and spectral methods [2-4]. Surrogate-based methods approximate a full-order model by a surrogate model, such as kriging. Spectral methods, such as proper orthogonal decomposition (POD), simplify a full-order model by identifying the dominating components of the response. The combination of surrogate-based methods and spectral methods, i.e. the POD-kriging method, has been demonstrated to be effective in accelerating the fluid solver in computational hypersonic aerothermoelasticity [2]. The POD-kriging method has been applied to aerodynamic loading and heating modeling of a 3D control surface [3-5] and skin panels [2, 6]. As illustrated in Refs. [5, 6], the performance of the fluid solver can be accelerated by a factor of 1000-10000, while retaining the accuracy of a Navier-Stokes solver.

Aeroelastic and aerothermoelastic behavior of skin panels of high speed missiles has been studied extensively, as shown in a comprehensive review [7]. Recently, a fully-coupled fluid-thermal-structural interactions (FTSI) computational framework was used to simulate the long-time-scale behavior of skin panels in hypersonic flow [6]. However, these studies focus on the response of a flat panel that is aligned to flow direction, which may not represent actual operating conditions. On the surface of a generic hypersonic vehicle, the panel is frequently curved. Furthermore, the direction of the flow will rarely coincide with that of the panel, an effect that can have considerable influence on dynamical behavior of the panel, as shown in [8]. None of the studies addressed the problem using a fully-coupled FTSI approach.

In current study, the authors described the acceleration of the computational framework named HYPATE, HYPersonic AeroThermoElasticity simulation environment [9]. The framework is used to study panel flutter problems in hypersonic flow, with emphasis on the effect of flow orientation angle and turbulent boundary layer thickness.

The remainder of this paper is arranged as follows: Section II details the MOR techniques for accelerating the HYPATE framework. Section III presents the verification of the computational framework and its components, particularly the ROM of the fluid solver. Section IV presents results on the application of HYPATE to panel flutter problems. Finally, section $\mathrm{V}$ provides concluding remarks. 


\section{Model Order Reduction of the Framework}

\section{A. Overview of the Computational Framework}

In Ref. [9], the HYPATE framework has been developed for time-accurate FTSI simulation. The HYPATE framework combines the fluid, structural, and thermal solvers using a second-order time accurate loosely-coupled scheme. Recently, the framework has been extended to include three fluid solvers having different levels of fidelity; meanwhile, the structural and thermal solvers are accelerated by parallelization. The code structure of the extended HYPATE framework is shown in Fig. 1.

The structural and thermal solvers are based on a C++ library with Python interface developed for general finite element (FE) analysis [9]. The structural solver is capable of modeling the structural dynamics of anisotropic doubly curved shallow shells, with shear, geometric nonlinearity, and thermal stress. The thermal solver models heat transfer in composite shells using layer-wise thermal lamination theory. Both solvers account for temperature-dependent material properties. The FE library is parallelized using the PETSc linear algebra library [10], which shows excellent scalability. The acceleration is illustrated in Fig. 2, where the computational costs of the solvers in original framework are used as reference. The structural and thermal solvers are both accelerated by two orders of magnitude.

The low-fidelity fluid solver is based on analytical models. The aerodynamic pressure is computed using linearized supersonic theory for supersonic flow, and piston theory for hypersonic flow. The heat flux is computed using Eckert's reference method. The low-fidelity fluid solver is used to verify the correctness of the code implementation of the framework. The next level up is a ROM-based fluid solver that has higher fidelity. The ROM-based fluid solver is the primary means for the reduction of computational cost in the hypersonic aerothermoelastic simulation. The ROM technique will be described in detail in the following sections. The high-fidelity fluid solver is the CFD solver. Recently, the previous CFD solver, SUmb, was upgraded by its successor, ADflow (Automatic Differentiation flow solver).

The new CFD solver introduces the restart capability, which is important for both steady/unsteady hypersonic simulations. In hypersonic regime, if the flow solution is initialized uniformly using the free stream condition, the simulation takes thousands of iterations to converge, i.e. to develop boundary layers and shock structures. When the solver is restarted, the flow simulation is initialized with a converged flow solution. Therefore, the restarted simulation is already nearly convergent, and the computational cost is reduced significantly. Furthermore, when the solution is nearly convergent, the solver is numerically sufficiently stable, so that other acceleration techniques, such as the multigrid method [11], can be applied to accelerate the convergence. The restart capability is used extensively for sample generation in the fluid ROM, described in Section II.

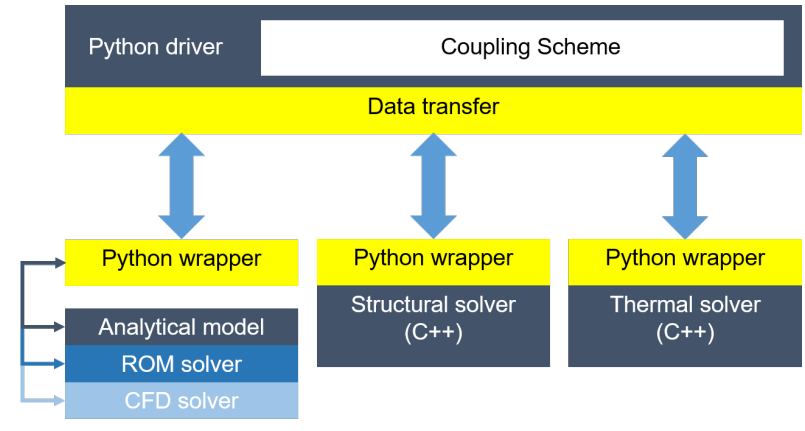

Figure 1. The code structure of the extended HYPATE framework.

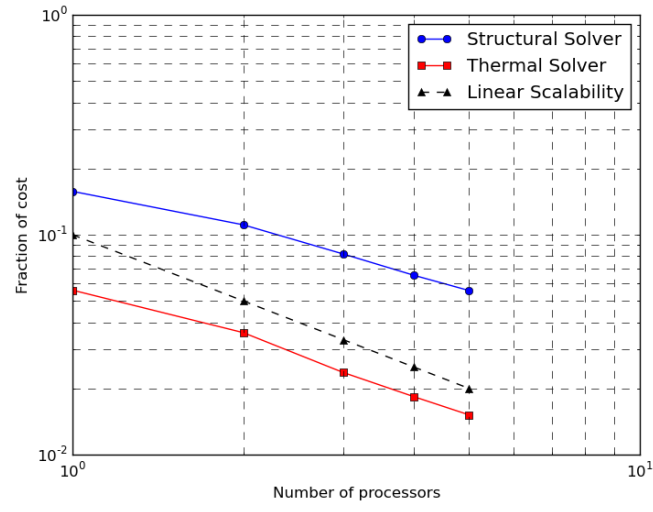

Figure 2. Parallel scalability of the finite element library.

\section{B. The POD-Kriging Model}

A fluid ROM usually relies on some simplifications of the flow physics, so as to reduce the computational cost for ROM generation. Therefore, a practical ROM usually involves in two components. The first component is an interpolation model for fluid solutions with simplified flow physics. The second is a correction model to compensate for the error introduced by the simplifications.

The key assumptions employed in the fluid ROM are,

1. Thermal solution of the structure is independent of structural deformation. 
2. The fluid solution is quasi-steady.

The first assumption is appropriate when structural deformation is small to moderate. It is justified mathematically in Ref. [9] for skin panels. The second assumption is due to the disparity of characteristic times of different physical domains. The fluid characteristic time is several orders of magnitude smaller than the structural characteristic time, and the structural characteristic time is several orders of magnitude smaller than the thermal characteristic time [12]. The second assumption leads to two approximations,

1. The pressure is weakly coupled with structural velocity.

2. The surface heat flux is independent of any terms associated with time derivatives, including structural velocity.

In the following, two common techniques for generating interpolation models are described, i.e. kriging method and proper orthogonal decomposition (POD) method. Then, the modeling considerations for pressure are discussed. Finally, the fluid ROM and its error analysis are presented.

\section{Kriging model}

The key assumption of kriging method is that two sample points that are close to each other in the parameter space have similar errors. This assumption is feasible for an unknown black-box function defined by deterministic computer simulations, since no sources of random error exists in the sample data set [13-15].

It suffices to consider kriging model for a function with vector input and scalar output. For a function with vector output, a kriging model can be constructed for each dimension of the output. With a given sample data set $\mathcal{D}=$ $\left\{\mathbf{x}_{i}, y_{i}\right\}_{i=1}^{m}$, the kriging interpolation is characterized by a global approximation $f$ corrected using local deviations $Z$,

$$
y(\mathbf{x})=f(\mathbf{x})+Z(\mathbf{x})
$$

The global approximation $f$ is a regression function representing the global trend of the unknown function,

$$
f(\mathbf{x})=\mathbf{f}(\mathbf{x})^{T} \boldsymbol{\beta}=\sum_{i=1}^{q} \beta_{i} f_{i}(\mathbf{x})
$$

In practice, the basis functions $\mathbf{f}(\mathbf{x})$ are polynomials up to second-order. When the basis function is zeroth-order, i.e. the global trend is a constant value, the kriging model is usually referred to as "ordinary kriging". Ordinary kriging has been applied widely in engineering designs, such as Ref. [15]. Linear and quadratic basis functions have been used in kriging models for fluid ROMs, such as Refs. [3, 5]. The results show that kriging model with quadratic basis functions generates more accurate results than the one with linear basis functions.

The local deviation $Z$ is assumed to resemble the behavior of a Gaussian process with zero mean and variance of $\sigma_{k r g}^{2}$. Using a correlation function $R\left(\mathbf{x}_{i}, \mathbf{x}_{j}\right)$, the covariance between two sample points is,

$$
\operatorname{cov}\left[Z\left(\mathbf{x}_{i}, \mathbf{x}_{j}\right)\right]=\sigma_{k r g}^{2} R\left(\mathbf{x}_{i}, \mathbf{x}_{j}\right)
$$

The correlation function measures the "closeness" between two points: The function value decreases as $\left\|\mathbf{x}_{i}-\mathbf{x}_{j}\right\|$ increases. A typical choice of the correlation function is the Gaussian kernel,

$$
R\left(\mathbf{x}_{i}, \mathbf{x}_{j}\right)=\exp \left[-\left(\mathbf{x}_{i}-\mathbf{x}_{j}\right)^{T} \mathbf{\Theta}\left(\mathbf{x}_{i}-\mathbf{x}_{j}\right)\right]
$$

The kriging model of the black-box function and the expectation of error are [13],

$$
\begin{aligned}
\hat{y}(\mathbf{x}) & =\mathbf{f}(\mathbf{x})^{T} \boldsymbol{\beta}+\mathbf{r}(\mathbf{x})^{T} \mathbf{R}^{-1}(\mathbf{y}-\mathbf{F} \boldsymbol{\beta}) \\
\epsilon_{k r g} & =\sigma_{k r g}^{2}\left[1+\mathbf{t}(\mathbf{x})^{T}\left(\mathbf{F}^{T} \mathbf{R}^{-1} \mathbf{F}\right)^{-1} \mathbf{t}(\mathbf{x})-\mathbf{r}(\mathbf{x})^{T} \mathbf{R}^{-1} \mathbf{r}(\mathbf{x})\right]
\end{aligned}
$$

where,

$$
\begin{aligned}
{[\mathbf{F}]_{i j}=f_{i}\left(\mathbf{x}_{j}\right), } & {[\mathbf{R}]_{i j}=R\left(\mathbf{x}_{i}, \mathbf{x}_{j}\right), } \\
\mathbf{r}(\mathbf{x})=\left[R\left(\mathbf{x}, \mathbf{x}_{1}\right), \cdots, R\left(\mathbf{x}, \mathbf{x}_{m}\right)\right], & \mathbf{t}(\mathbf{x})=\mathbf{F}^{T} \mathbf{R}^{-1} \mathbf{r}(\mathbf{x})-\mathbf{f}(\mathbf{x})
\end{aligned}
$$

\section{Proper Orthogonal Decomposition}

The POD method has been used for dimension reduction problems [16, 17]. It provides a means to reproduce the behavior of a full-order system using a lower-order model, and thus significantly reduces computational cost. The low-order model is constructed from a few "POD modes", which represent the dominating patterns in the responses 
of a full-order system. Reference [16] is the first study to apply POD in fluid problems. Reference [17] provides a detailed description of the POD method in general.

For fluid dynamics, the POD modes are usually constructed using the snapshot method [16]. Consider a sample data set of full-order solutions $\left\{\mathbf{y}_{i}\right\}_{i=1}^{m}$, the snapshot matrix is,

$$
\mathbf{S}=\left[\mathbf{y}_{1}, \cdots, \mathbf{y}_{m}\right]
$$

Take the singular value decomposition (SVD) of the snapshot matrix,

$$
\mathbf{S}=\mathbf{U} \boldsymbol{\Sigma} \mathbf{V}^{T}
$$

where $\mathbf{U}$ and $\mathbf{V}$ are orthonormal matrices, whose columns are the left and right singular vectors of $\mathbf{S}$, respectively. The matrix $\boldsymbol{\Sigma}$ is diagonal, and the nonzero entries are singular values of $\mathbf{S}$ in decreasing order.

Let the first $r$ left singular vectors be the POD modes,

$$
\boldsymbol{\Psi}=\left[\mathbf{u}_{1}, \cdots, \mathbf{u}_{r}\right]
$$

The snapshot matrix is approximated by projection onto the space spanned by $\Psi$,

$$
\mathbf{S} \approx \boldsymbol{\Psi} \boldsymbol{\Psi}^{T} \mathbf{S}
$$

The error of approximation is,

$$
\left\|\boldsymbol{\epsilon}_{P O D}\right\|^{2}=\left\|\mathbf{S}-\boldsymbol{\Psi} \Psi^{T} \mathbf{S}\right\|^{2}=\sum_{i=r+1}^{m} \sigma_{i}^{2}
$$

A full-order solution $\mathbf{y}$ and its POD components $\mathbf{y}_{P}$ are related by POD transformation,

$$
\mathbf{y}_{P}=\Psi^{T} \mathbf{y}, \quad \mathbf{y} \approx \mathbf{\Psi} \mathbf{y}_{P}
$$

\section{Modeling considerations for unsteady pressure}

Due to the quasi-steady assumption of the fluid ROM, given surface deformation and velocity, the pressure $C_{p}(w, \dot{w})$ is decomposed into the steady and unsteady components.

$$
C_{p}(w, \dot{w})=C_{p}^{s t d}(w)+C_{p}^{u n s}(w, \dot{w})
$$

where the steady component $C_{p}^{\text {std }}$ is the pressure when the surface is statically deformed. The steady component is computed using a CFD solver or a fluid ROM. The unsteady correction for pressure is developed from analytical inviscid flow solutions.

In hypersonic flow regime, the inviscid flow can be approximated by piston theory [18]. Its third order expansion is,

$$
\begin{aligned}
C_{p}(w, \dot{w}) & =C_{p, d e f}^{p t}(w)+C_{p, \text { mix }}^{p t}(w, \dot{w})+C_{p, v e l}^{p t}(w, \dot{w}) \\
C_{p, d e f}^{p t}(w) & =\frac{2}{M_{\infty}^{2}}\left(M_{\infty} w_{, x}\right)+\frac{1+\gamma}{2 M_{\infty}^{2}}\left(M_{\infty} w_{, x}\right)^{2}+\frac{1+\gamma}{6 M_{\infty}^{2}}\left(M_{\infty} w_{, x}\right)^{3} \\
C_{p, \text { mix }}^{p t}(w, \dot{w}) & =\frac{1+\gamma}{2 M_{\infty}^{2}} \frac{\dot{w}}{a_{\infty}}\left(M_{\infty} w_{, x}\right)\left(2+\frac{\dot{w}}{a_{\infty}}+M_{\infty} w_{, x}\right) \\
C_{p, v e l}^{p t}(w, \dot{w}) & =\frac{2}{M_{\infty}^{2}} \frac{\dot{w}}{a_{\infty}}+\frac{1+\gamma}{2 M_{\infty}^{2}}\left(\frac{\dot{w}}{a_{\infty}}\right)^{2}+\frac{1+\gamma}{6 M_{\infty}^{2}}\left(\frac{\dot{w}}{a_{\infty}}\right)^{3}
\end{aligned}
$$

where $C_{p, d e f}$ is the pressure component due to deformation only, $C_{p, \text { mix }}$ is the pressure component due to deformation and velocity, $C_{p, v e l}$ is the pressure component due to velocity only. The term $C_{p}^{s t d}(w)$ in Eq. (13) is essentially the viscous counterpart for $C_{p, d e f}^{p t}(w)$ in Eq. (14). The other terms in Eq. (14), which are velocity-dependent, are combined to obtain unsteady correction [5],

$$
C_{p}^{u n s, p t}(w, \dot{w})=C_{p, m i x}^{p t}(w, \dot{w})+C_{p, v e l}^{p t}(w, \dot{w})
$$


In supersonic flow regime, inviscid pressure is approximated by linearized supersonic theory (LnSp) [19],

$$
\begin{aligned}
C_{p}(w, \dot{w}) & =C_{p, d e f}^{\text {lnsp }}(w)+C_{p, v e l}^{\text {lnsp }}(w, \dot{w}) \\
C_{p, d e f}^{\text {lnsp }}(w) & =\frac{2}{\sqrt{M_{\infty}^{2}-1}} w_{, x} \\
C_{p, \text { vel }}^{\text {lnsp }}(w, \dot{w}) & =\frac{2}{\sqrt{M_{\infty}^{2}-1}} \frac{M_{\infty}^{2}-2}{M_{\infty}^{2}-1} \frac{\dot{w}}{U_{\infty}}
\end{aligned}
$$

Similar to the treatment in piston theory, with assumption 3, the velocity-dependent component is used for unsteady correction,

$$
C_{p}^{u n s, l n s p}(w, \dot{w})=C_{p, v e l}^{l n s p}(w, \dot{w})
$$

\section{The fluid ROM using POD-kriging method}

The quantities of interest in the fluid solver are the unsteady aerodynamic loading $p$ and heating on the deforming structure $\dot{q}$. For more flexibility in the ROM, the pressure coefficient and the Stanton number are preferred over their dimensioned counterparts. These quantities are represented as vectors,

$$
\begin{aligned}
& \mathbf{p}=\left[C_{p}^{(1)}, \cdots, C_{p}^{(n)}\right] \\
& \dot{\mathbf{q}}=\left[C_{H}^{(1)}, \cdots, C_{H}^{(n)}\right]
\end{aligned}
$$

where $C_{p}^{(i)}, C_{H}^{(i)}$ are the pressure coefficient and Stanton number at the $i$ th node of the mesh.

Following the discussion in previous section, the aerodynamic loading is decomposed into two parts, the steady component $\mathbf{p}_{s t d}$ and the unsteady correction $\mathbf{p}_{\text {uns }}$. The POD-kriging method is used to model the steady component of the aerodynamic loading. The unsteady component is computed using analytical relations.

The fluid solutions depends on a set parameters $\mathbf{x}$. The parameters typically characterize flight conditions $\mathbf{c}$, structural deformation $w$, and wall temperature $T_{w}$. For a skin panel, a possible combination of flight conditions is $\mathbf{c}=\left[M_{\infty}, R e, \beta\right]$. The structural deformation is modeled using multiple structural modes, and the wall temperature is approximated using polynomial functions,

$$
\begin{aligned}
w(x, y) & =\sum_{i=1}^{n_{S}} a_{i} \psi_{S i}(x, y) \\
T_{w}(x, y) & =\sum_{i=1}^{n_{T}} b_{i} \psi_{T i}(x, y)
\end{aligned}
$$

Then, the fluid solution of a skin panel depends on a vector of $\left(n_{F}+n_{S}+n_{T}\right)$ parameters,

$$
\mathbf{x}=\left[M_{\infty}, R e, \beta, \cdots, a_{1}, \cdots, a_{n_{S}}, b_{1}, \cdots, b_{n_{T}}\right]
$$

The computational cost for prediction using kriging model is proportional to the dimension of the output. If the kriging model relates the parameter vector to the fluid solutions directly, dimension of the output is of the order of $10^{4}$. However, the fluid solution can be approximated by a few POD modes (typically less than 50). In the POD-kriging method, the output of the kriging model is the POD components, instead of the whole solution, the computational cost of kriging model can be reduced by an order of $10^{3}$.

The procedure for the generation and application of the POD-kriging model are illustrated in Fig. 3.

1. Generate a set of sample points $\left\{\mathbf{x}_{i}\right\}_{i=1}^{m}$ using optimal latin hypercube (OLH) sampling method.

2. Generate high-fidelity steady solutions $\left\{\mathbf{p}_{s t d, i}\right\}_{i=1}^{m},\left\{\dot{\mathbf{q}}_{i}\right\}_{i=1}^{m}$ for each sample point.

3. Apply POD to the sample solutions, and approximate the fluid solutions by,

$$
\mathbf{p}_{P i}=\Psi_{p}^{T} \mathbf{p}_{s t d, i}, \quad \dot{\mathbf{q}}_{P i}=\mathbf{\Psi}_{q}^{T} \dot{\mathbf{q}}_{i}
$$

where the columns of $\Psi_{p}, \Psi_{q}$ are POD modes for distributions of pressure and heat flux, respectively. Then denote,

$$
\mathbf{y}_{P i}=\left[\mathbf{p}_{P i}, \dot{\mathbf{q}}_{P i}\right]
$$

4. Train a kriging model $\mathbf{y}_{P O D-k r g}$ using the data set $\left\{\mathbf{x}_{i}, \mathbf{y}_{P i}\right\}_{i=1}^{m}$. 
The procedure for prediction using POD-kriging model involves three steps. Given a new parameter vector $\mathbf{x}$,

1. Obtain the POD components of the fluid solutions,

$$
\hat{\mathbf{y}}_{P}=\left[\hat{\mathbf{p}}_{P}, \hat{\dot{\mathbf{q}}}_{P}\right]=\mathbf{y}_{P O D-k r g}(\hat{\mathbf{x}})
$$

2. Recover the fluid solution by POD transformation,

$$
\hat{\mathbf{p}}_{s t d}=\boldsymbol{\Psi}_{p} \hat{\mathbf{p}}_{P}, \quad \hat{\dot{\mathbf{q}}}=\boldsymbol{\Psi}_{q} \hat{\dot{\mathbf{q}}}_{P}
$$

3. Correct the pressure for unsteadiness,

$$
\hat{\mathbf{p}}=\hat{\mathbf{p}}_{s t d}+\mathbf{p}_{u n s}(w, \dot{w})
$$

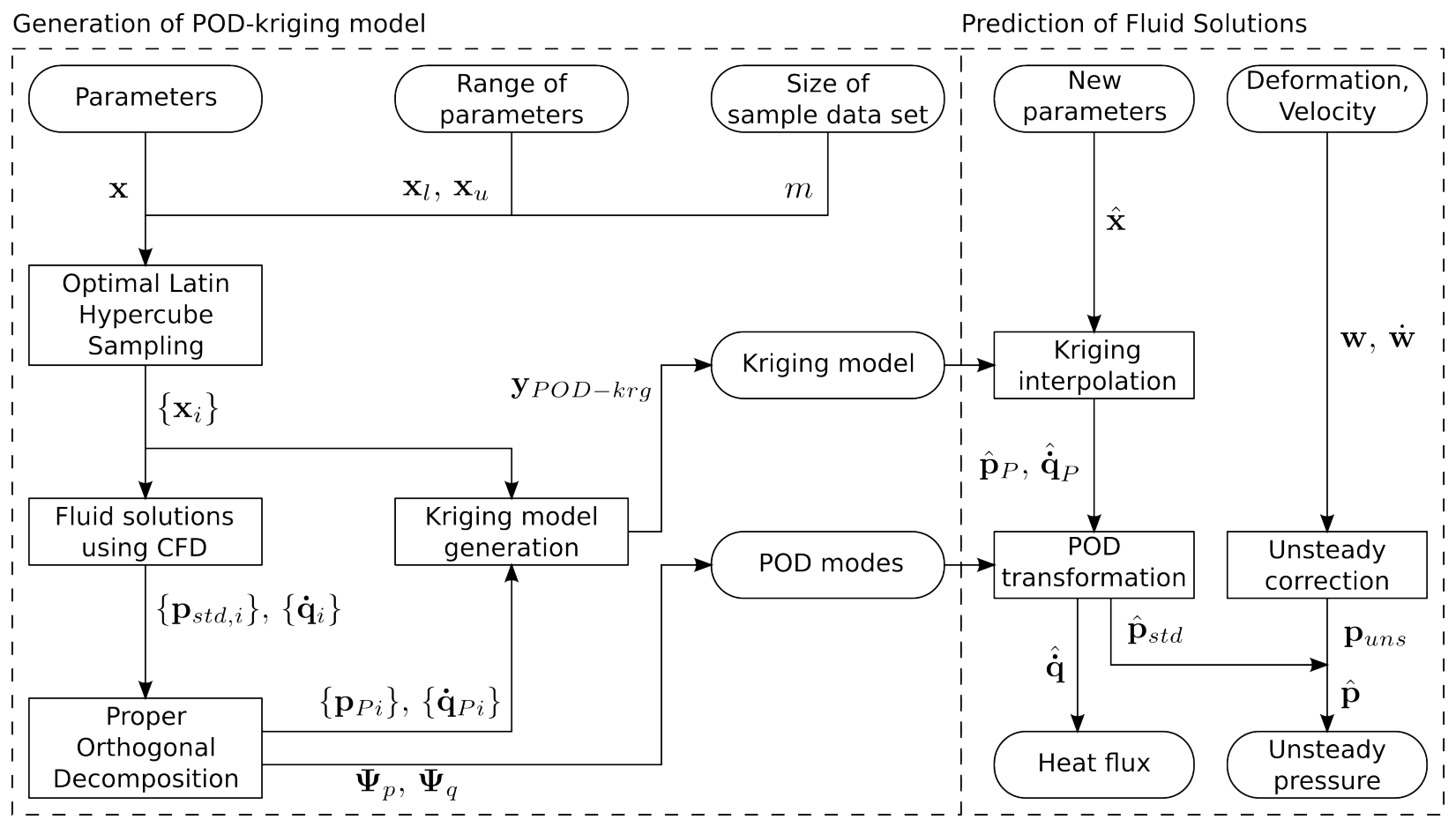

Figure 3. The generation and application of the fluid ROM.

\section{Error analysis of the fluid ROM}

The unsteady aerodynamic loading $\mathbf{p}$ and heat flux $\dot{\mathbf{q}}$ depend on flight conditions $\mathbf{c}$, surface deformation $w$, and surface temperature $T_{w}$. The relation is denoted symbolically as,

$$
\mathbf{p}=\mathbf{p}\left(\mathbf{c}, w, T_{w}\right), \quad \dot{\mathbf{q}}=\dot{\mathbf{q}}\left(\mathbf{c}, w, T_{w}\right)
$$

In the fluid ROM, Eq. (33) is approximated using the POD-kriging model and unsteady correction.

$$
\begin{aligned}
\mathbf{p} & =\mathbf{p}_{s t d}(\mathbf{c}, \mathbf{a}, \mathbf{b})+\mathbf{p}_{\text {uns }}\left(\mathbf{c}, w, T_{w}\right)+\boldsymbol{\epsilon}_{\text {pod }}+\boldsymbol{\epsilon}_{k r g}+\boldsymbol{\epsilon}_{\text {uns }}+\boldsymbol{\epsilon}_{m o d} \\
& \approx \mathbf{p}_{s t d}(\mathbf{c}, \mathbf{a}, \mathbf{b})+\mathbf{p}_{\text {uns }}\left(\mathbf{c}, w, T_{w}\right) \\
\dot{\mathbf{q}} & =\dot{\mathbf{q}}_{s t d}(\mathbf{c}, \mathbf{a}, \mathbf{b})+\boldsymbol{\epsilon}_{p o d}+\boldsymbol{\epsilon}_{k r g}+\boldsymbol{\epsilon}_{m o d} \\
& \approx \dot{\mathbf{q}}_{s t d}(\mathbf{c}, \mathbf{a}, \mathbf{b})
\end{aligned}
$$

The approximation introduces four types of error:

1. $\epsilon_{p o d}$ : The approximation of fluid solution using POD transformation.

2. $\epsilon_{k r g}$ : The uncertainty in the kriging model.

3. $\epsilon_{\text {uns }}$ : The analytical correction for unsteady component. This term only exists in the pressure prediction.

4. $\epsilon_{m o d}$ : The representation of deformation and temperature using shape functions. 
If the parameter space is well represented by the sample data set, the error of the ROM is bounded by,

$$
\|\boldsymbol{\epsilon}\| \leq\left\|\boldsymbol{\epsilon}_{c o r}\right\|+\left\|\boldsymbol{\epsilon}_{m o d}\right\|+\left\|\boldsymbol{\epsilon}_{\text {pod }}\right\|+\left\|\boldsymbol{\epsilon}_{k r g}\right\|
$$

The error due to POD is determined through SVD using Eq. (11). The error due to kriging at a given parameter combination can be determined using the probabilistic interpretation of the kriging model, i.e. Eq. (6). The error due to correction can be only estimated empirically. The error due to modal approximation cannot be determined directly, but can be estimated by,

$$
\left\|\boldsymbol{\epsilon}_{m o d}\right\| \leq E_{1}\left\|w-\sum_{i=1}^{n_{S}} a_{i} \psi_{S i}\right\|+E_{2}\left\|T_{w}-\sum_{i=1}^{n_{T}} b_{i} \psi_{T i}\right\|
$$

where $E_{1}, E_{2}$ are empirical constants to be determined numerically.

\section{The Smart Ordering Scheme}

Over $95 \%$ of the time for generating a fluid ROM is consumed by the computation of the sample fluid solutions. The smart ordering scheme accelerates the computation of the samples, and reduces the computational cost of ROM generation by a factor of 2 .

The scheme utilizes the "restart" capability of the CFD solver. The fluid simulation is initialized using a reference solution $u^{*}$, and converges to the actual solution $u$ after a certain number of iterations. When the reference solution is similar to the actual solution, the solver converges in fewer iterations and less time. The smart ordering scheme initializes a sample solution $u$ using another sample solution $u^{*}$ that is the most similar to $u$, so that the computational cost of $u$ is minimized. The similarity between the reference solution $u^{*}$ and the actual solution $u$ is characterized by the "distance" between the two fluid solutions.

Similar to Eq. (33), the fluid solution depends on the flight conditions, structural deformation, and wall temperature,

$$
u=u\left(\mathbf{c}, w, T_{w}\right)
$$

Assuming that the computational cost of the fluid solution is proportional to the difference between the reference and actual solutions, one has,

$$
\begin{aligned}
t_{C F D}(u) & \propto\left\|u-u^{*}\right\| \\
& =\left\|u\left(\mathbf{c}, w, T_{w}\right)-u\left(\mathbf{c}^{*}, w^{*}, T_{w}^{*}\right)\right\| \\
& \leq C_{1}\left\|\mathbf{c}-\mathbf{c}^{*}\right\|+C_{2}\left\|w-w^{*}\right\|+C_{3}\left\|T_{w}-T_{w}^{*}\right\|
\end{aligned}
$$

where $C_{1}, C_{2}, C_{3}$ are constants due to Taylor expansion of $u\left(\mathbf{c}, w, T_{w}\right)$.

In the POD-kriging ROM, the structural deformation and wall temperature are represented using shape functions in Eqns. (25)-(26). Assuming the shape functions are orthogonal and normalized,

$$
\begin{array}{r}
\left\|w-w^{*}\right\|=\left\|\sum_{i=1}^{n_{S}} a_{i} \psi_{S i}-\sum_{i=1}^{n_{S}} a_{i}^{*} \psi_{S i}\right\|=\left\|\mathbf{a}-\mathbf{a}^{*}\right\| \\
\left\|T_{w}-T_{w}^{*}\right\|=\left\|\sum_{i=1}^{n_{T}} b_{i} \psi_{T i}-\sum_{i=1}^{n_{T}} b_{i}^{*} \psi_{T i}\right\|=\left\|\mathbf{b}-\mathbf{b}^{*}\right\|
\end{array}
$$

Combining equations (37)-(39), the distance between two fluid solutions is defined as,

$$
d\left(u, u^{*}\right)=\sqrt{\sum_{i=1}^{n_{F}} D_{1 i}\left(c_{i}-c_{i}^{*}\right)^{2}}+D_{2}\left\|\mathbf{a}-\mathbf{a}^{*}\right\|+D_{3}\left\|\mathbf{b}-\mathbf{b}^{*}\right\|
$$

where $D_{1 i}, D_{2}, D_{3}$ are empirical constants for nondimensionalization, so that the quantities with different units, such as the deformation, temperature, and flow angles, can be summed up.

The total computational cost of computing the ROM samples $\mathcal{D}$ is proportional to the sum of the distances between the fluid solutions and their reference solutions.

$$
t_{C F D}(\mathcal{D}) \propto \sum_{i=1}^{N_{s}} d\left(u_{i}, u_{i}^{*}\right)
$$


The reference solution $u_{i}^{*}$ can be a naive fluid solution $u_{0}$ representing the flow field with undeformed panel and uniform wall temperature. The naive fluid solution is computed before the ROM generation. The reference solution can also be one of the sample fluid solutions. The best reference solution $u_{i}^{*}$ is the one that is the closest to the $i$ th sample $u_{i}$, so that the computation cost of $u_{i}$ is minimized. In conventional ROM generation, the samples are always restarted using the naive fluid solution $u_{0}$.

The algorithm for smart ordering scheme is presented in the Appendix A. The samples are ordered such that:

1. The first sample solution is restarted using the naive fluid solution $u_{0}$.

2. If the $i$ th sample $u_{i}$ is the reference solution for the $j$ th sample $u_{j}$, then $d\left(u_{i}, u_{j}\right)$ is minimum, and $u_{i}$ is always computed before $u_{j}$.

3. Multiple samples might use the same sample solution as reference solution.

The comparison of the computational cost using the conventional method and the smart ordering scheme is shown in Table 1. For simplicity, the fluid solution is described using 3 structural modes only, i.e. $n_{F}=0, n_{S}=3, n_{T}=0$. One hundred 3D solutions are generated. Using smart ordering scheme, the sample generation is accelerated by approximately two times.

Table 1. Computational cost in sample generation.

\begin{tabular}{cccc}
\hline & Conventional & Smart ordering & Acceleration \\
\hline Euler & $1.2 \mathrm{hrs}$ & $0.7 \mathrm{hrs}$ & 1.7 \\
RANS & $2 \mathrm{hrs}$ & $0.9 \mathrm{hrs}$ & 2.2 \\
\hline
\end{tabular}

\section{Verification of the Framework}

\section{A. Two-Dimensional Panel Flutter}

\section{Case Description}

First, the framework is verified for a semi-infinite panel, i.e. a 2D panel. The configuration considered is taken from Ref. [20], where inviscid and viscous (laminar) tightly-coupled panel flutter simulations were performed at transonicsupersonic Mach numbers. The panel was simply supported on its leading and trailing edges, and had the following properties: thickness $h / a=0.002$, mass ratio $\mu_{s}=0.1$, Poisson's ratio $\nu=0.3$. The cases for comparison are listed in Table 2.

Table 2. Case parameters for $2 \mathrm{D}$ panel flutter

\begin{tabular}{ccccc}
\hline Case \# & Mach & Type & $\delta / a$ & $R e$ \\
\hline 1 & 1.2 & Euler & 0.0 & - \\
2 & 1.2 & Laminar & 0.025 & 100,000 \\
3 & 1.8 & Euler & 0.0 & - \\
\hline
\end{tabular}

\section{Solution Procedure}

The computational model for 2D panel is shown in Fig. 4. The structural mesh has 50 elements in streamwise direction and is one-element wide in spanwise direction. Symmetric BC are applied to the sides parallel to $x$ axis. The fluid domain is discretized using structured mesh. It consists of 170 points in the axial direction (65 of which are on the panel itself) and 81/105 points normal to the plate for the inviscid and viscous meshes respectively. The wall-normal spacing for the viscous mesh is $\Delta z / a=10^{-5}$ for a sufficient resolution of the boundary layer. The value of $l_{1}$ is adjusted to obtain the required boundary layer thickness over the panel. 


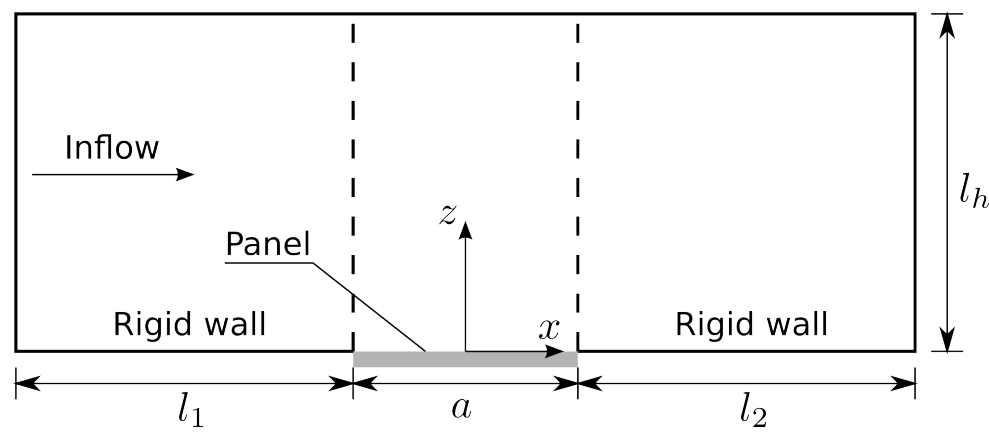

Figure 4. The computational model for $2 \mathrm{D}$ case.

The simulation is initialized as follows. First, a steady solution is obtained for the undeformed panel. The resulting pressure distribution due to boundary layer is applied below the panel in order to insure that the panel would oscillate around its undeformed shape. To initiate the unsteady flutter computation, an incremental deformation is prescribed by applying a small uniform load in $z$ direction. For all computations, a second-order accurate temporal integration scheme (dual time-stepping) for the flow solver is used with a time step of $\Delta t=0.5 a / U_{\infty}$. The time step is chosen after a limited time-step convergence study.

\section{Results}

The variation of flutter amplitude as a function of the non-dimensional dynamic pressure $\lambda$ at $x / a=0.75$ is shown in Fig. 5 for all cases. In addition to the comparison with the results from Ref. [20], the results at the higher Mach number $\left(M_{\infty}=1.8\right)$ are also compared with the linearized supersonic theory, which is applicable at Mach numbers above $\sqrt{2}[21]$.

All the cases show very good agreement. The delaying of the flutter onset and the reduction of amplitude growth rate due to the boundary layer are captured accurately, as well as the different flutter behaviours at transonic and supersonic Mach numbers. In addition, at the supersonic Mach number $\left(M_{\infty}=1.8\right)$ linearized supersonic theory provides very similar results to Euler computations, which means that the non-linearity is not very significant for this case.

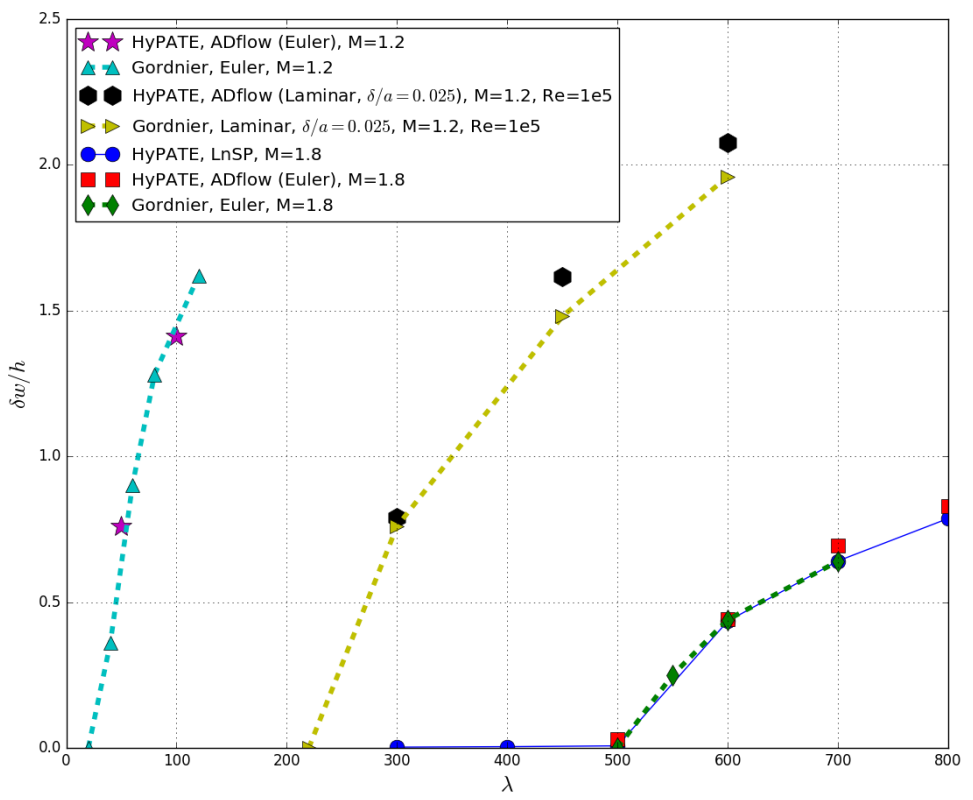

Figure 5. The LCO curves and comparison with Gordnier's results. 


\section{The fluid ROM for $2 D$ panel}

To verify the usage of ROM within HYPATE, a ROM was generated for $M_{\infty}=1.8$. This Mach number was chosen, since an unsteady correction can be obtained from LnSp (Eqn. 19). The ROM was generated using 3 structural (sine) modes with 50 samples. The unsteady ROM-coupled simulation is two orders of magnitude faster than the CFD-coupled solution.

The cumulative pressure POD energy for different number of samples is shown in Figure 6a. It can be seen that 30 samples are enough to achieve convergence in the mode energy distribution. Three modes contain the entire energy, while the first mode is the most energetic and contain $65 \%$ of the total energy, the second mode contain $30 \%$, and the third mode has the least energy with only $5 \%$. The computed pressure POD modes shapes are shown in Figure $6 \mathrm{~b}$. The first and third pressure mode shapes resemble to the first and second structural (sine) mode shapes, and represent combination of compression and expansion on the panel. The second pressure mode represent a gradual compression or expansion along the panel.

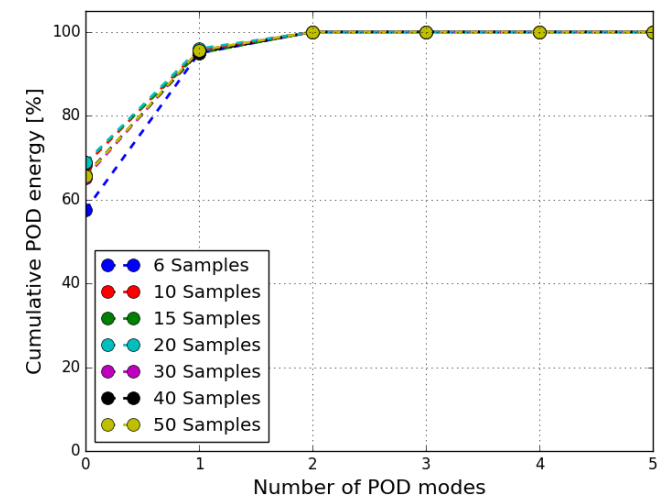

a) Cumulative POD energy

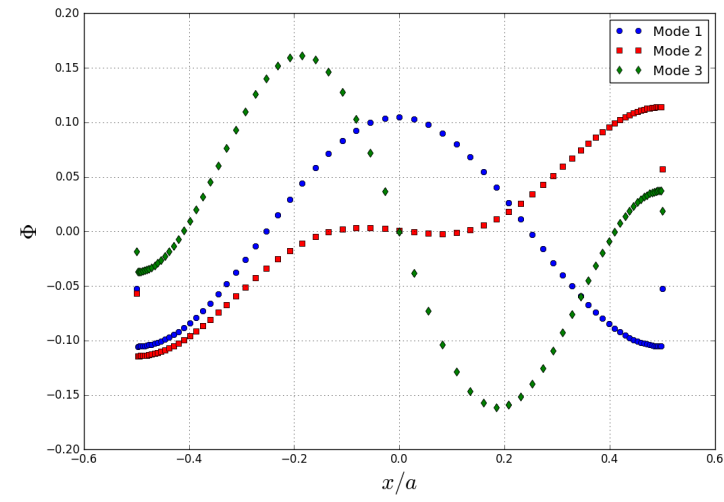

b) Pressure POD modes

Figure 6. Pressure POD for 2D panel at $M_{\infty}=1.8$.

The ROM was used to reproduce the unsteady flutter simulation results at different dynamic pressures. The comparison of the LCO curves is shown in Fig. 7. The agreement is good in general: the onset flutter dynamic pressure is captured accurately, and the amplitude growth rate is a little under-predicted. This is due to the fact that only three sine modes were used to represent the panel deformation.

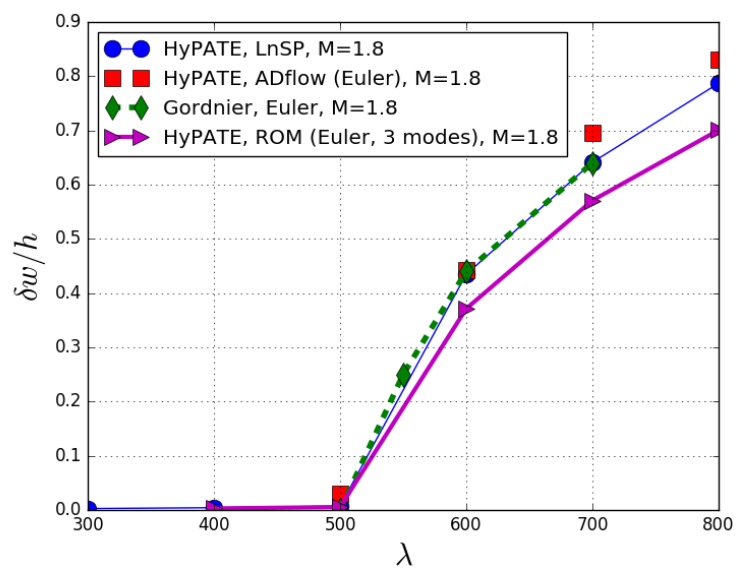

Figure 7. The LCO curves and comparison with ROM results. 


\section{B. Three-Dimensional Panel Flutter \\ 1. Case Description}

The configuration considered for 3D panel flutter is taken from Ref. [22], where a RANS-based tightly-coupled aeroelastic computational framework was developed and applied to panel flutter analysis over a range of $M_{\infty}=$ $1.1-2.4$. The geometric parameters and material properties of the panel are shown in Table 3. The panel is clamped along its four edges. The natural frequencies of the panel agree very well with data from literature, as shown in Table 4. The first 12 mode shapes are shown in Fig. 8.

Table 3. Panel properties for 3D panel flutter

\begin{tabular}{cccccc}
\hline$a$ & $b$ & $h$ & $\rho_{m}$ & $E$ & $\nu$ \\
\hline 9in & 18 in & $0.0401 \mathrm{in}$ & $110.0 \mathrm{lb} / \mathrm{ft}^{3}$ & $5.6 \mathrm{Mpsi}$ & 0.35 \\
\hline
\end{tabular}

Table 4. The natural frequencies of the panel

\begin{tabular}{cccccc}
\hline Mode No. & 1 & 2 & 3 & 4 & 5 \\
\hline Current & 110.0 & 142.4 & 200.3 & 283.4 & 286.4 \\
Ref. [22] & 108.1 & 138.2 & 191.7 & 267.9 & 278.4 \\
Difference (\%) & 1.71 & 3.04 & 4.49 & 5.79 & 2.87 \\
\hline
\end{tabular}

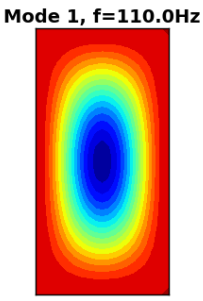

Mode 5, $\mathrm{f}=\mathbf{2 8 6 . 4 \mathrm { Hz }}$

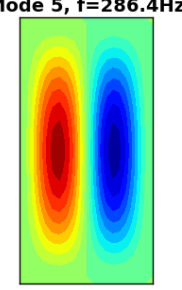

Mode 9, $f=451.0 H z$

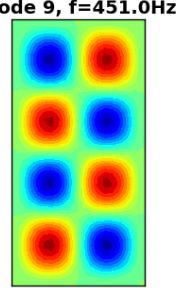

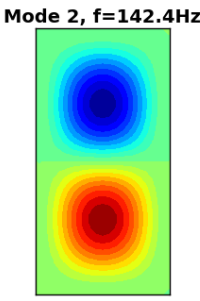

Mode 6, $f=318.1 \mathrm{~Hz}$

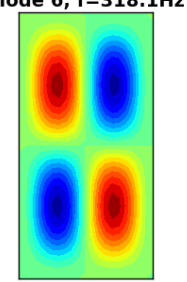

Mode 10, $f=521.3 \mathrm{~Hz}$

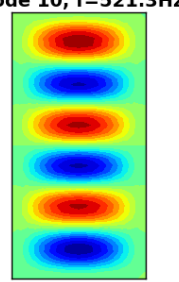

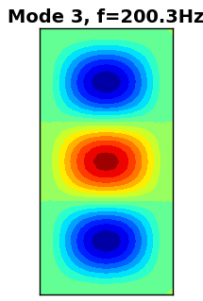

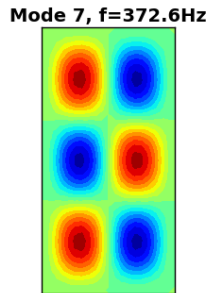

Mode 11, $f=552.7 \mathrm{~Hz}$

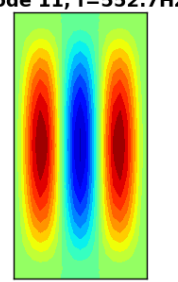

Mode 4, $\mathrm{f}=\mathbf{2 8 3 . 4 \mathrm { Hz }}$

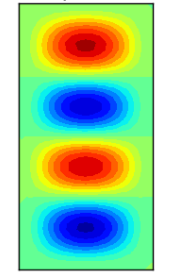

Mode 8, $f=390.6 \mathrm{~Hz}$

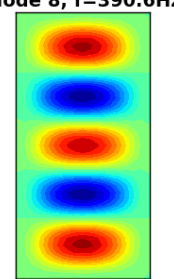

Mode 12, $\mathrm{f}=553.5 \mathrm{~Hz}$

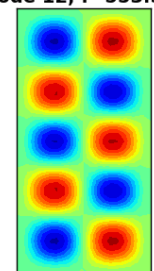

Figure 8. Mode shapes and frequencies for 3D panel, $b / a=2$.

\section{Results}

The CFD-coupled solution from HYPATE framework is verified using data from Ref. [22] in supersonic regime $\left(M_{\infty}=1.6-2.4\right)$. The computational model for the 3D panel is shown in Fig. 9. In the structural mesh, the panel is discretized using 10 elements in streamwise direction and 20 in spanwise direction. In the fluid mesh, the panel has 33 grid points in streamwise direction and 65 in spanwise direction. The $y^{+}$value of the mesh is kept under 1.0 for adequate resolution of the boundary layer. The value of $l_{1}$ is fixed, so that the boundary layer thickness at $x=0$ is $\delta / a=0.1$ at $M_{\infty}=2.4$. The initialization of the simulation is the same as that of the $2 \mathrm{D}$ case. 
The flutter boundary of the panel is shown in Fig. 10. The Euler and RANS results are shifted upward by approximately 5\%, compared with the data from Ref. [22]. The Euler and LnSp results agree well with each other. The error could be due to the fact that the structure used in current study is slightly stiffer than that in the reference. A stiffer panel is expected to have higher flutter boundary. For the RANS results, another source of error is the boundary layer. The values of $\delta / a$ in the reference and current study do not match exactly. The difference in boundary layer thickness may result in the shift of flutter boundary. The RANS and Euler results indicate that over Mach 1.9, the viscous effect stabilizes the panel, i.e. increases the flutter boundary. This trend is also confirmed by Ref. [22].

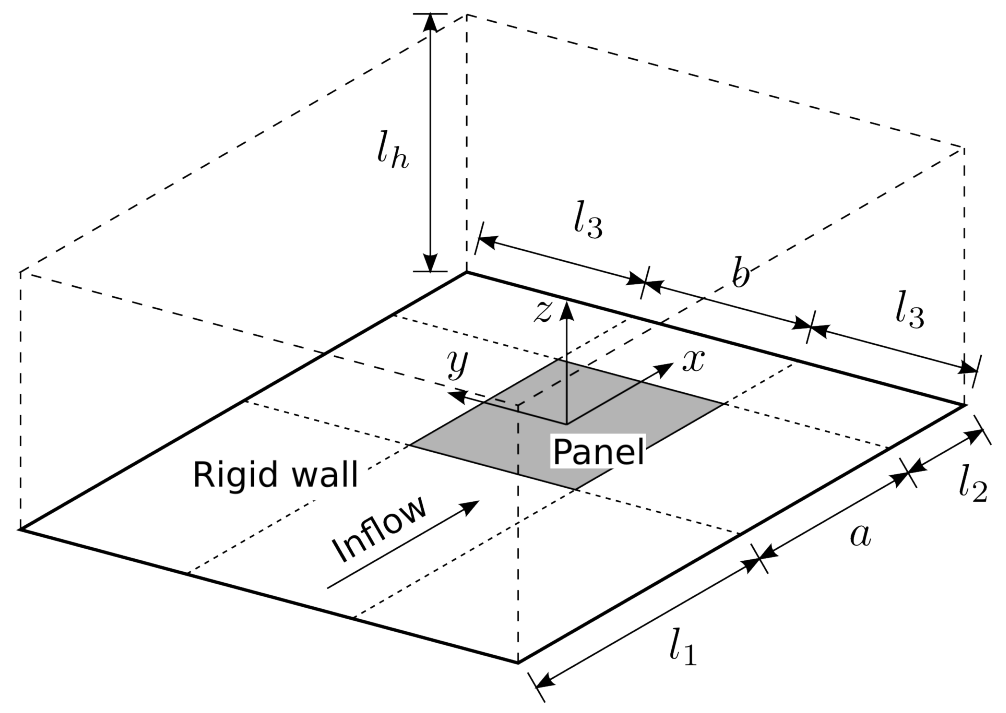

Figure 9. The computational model for 3D case.

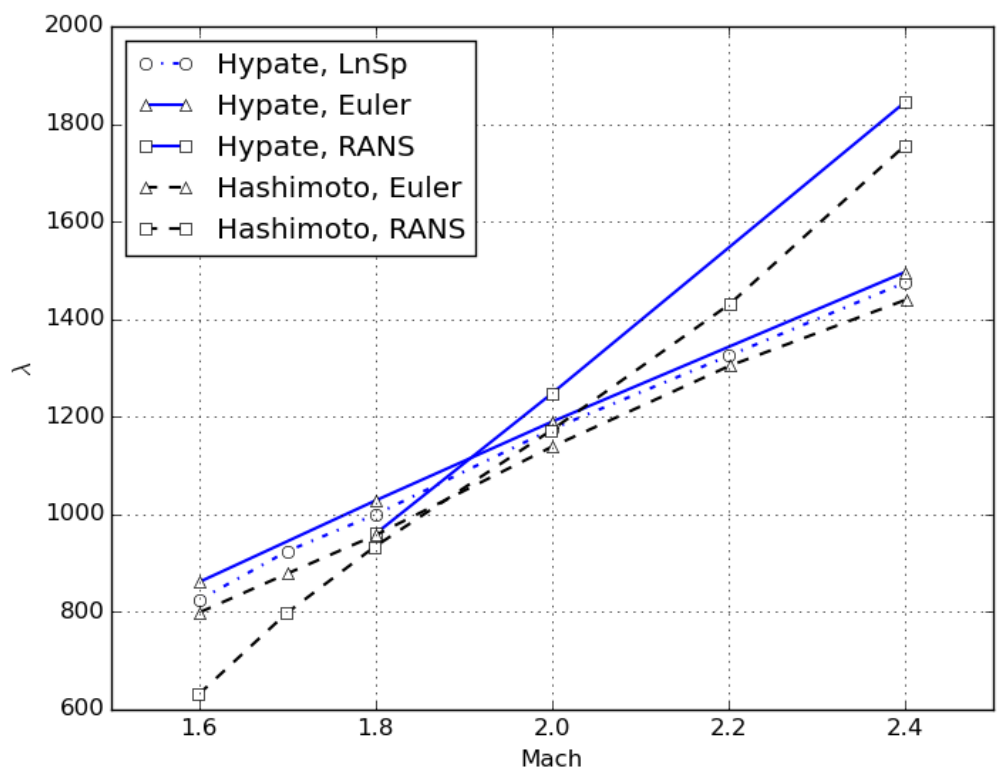

Figure 10. The flutter boundary for $M_{\infty}=1.6-2.4$. 


\section{Application to Hypersonic Panel Flutter}

\section{A. Case Description}

The flutter analysis for the 3D panel configuration from Ref. [22] is extended to hypersonic regime $\left(M_{\infty}=6\right)$. The HYPATE framework is applied to investigate the effects of boundary layer thickness $\delta / a$ and flow orientation angle $\beta$ on panel flutter.

To enable a similar boundary layer thickness at an arbitrary flow orientation angle, the fluid mesh in the computational model is switched to an O-grid mesh, as shown in Fig. 11. The distance of center of the panel to the inflow boundary is invariant, so the same boundary layer thickness at the panel is maintained for any orientation angles.

CFD-coupled solutions are generated for various values of dynamic pressures. These solutions are used to verify ROM-coupled solutions. The verified ROMs are used to produce the flutter boundary for various cases, which are presented next. The reduction in computer time is obtained due to the fluid ROM is illustrated in Table 5. In the comparison, the simulation lasts for 30 minutes with a time step of $0.4 \mathrm{~ms}$, and the computational costs are extrapolated from current data. The computational cost of fluid ROM is insensitive to the type of fluid equation (RANS/Euler). The fluid solver is accelerated by 5 orders of magnitude, and the coupled solution is accelerated by 3 orders of magnitude.

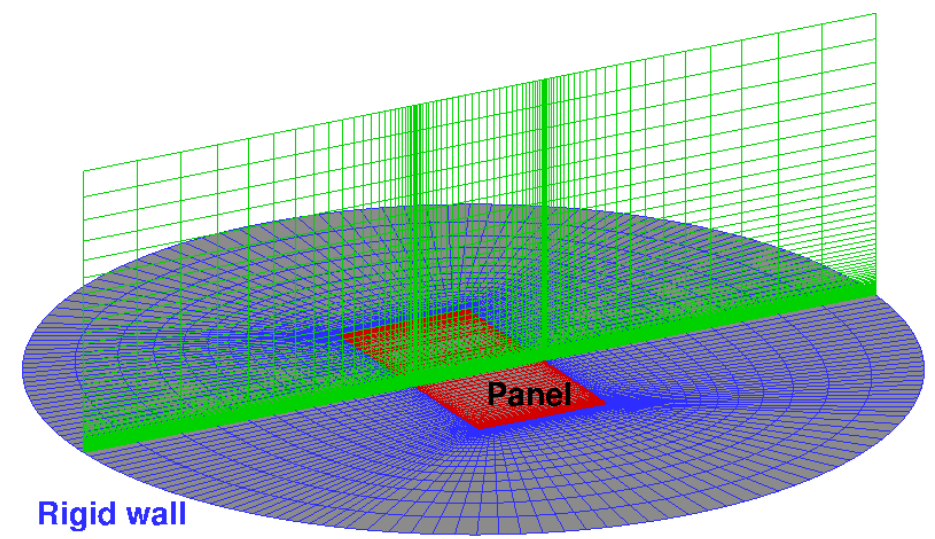

Figure 11. O-grid type mesh for 3D panel.

Table 5. Comparison of computational cost

\begin{tabular}{ccccc}
\hline 8 processors & Fluid solver & Structural solver & Data transfer & Cost $(30 \mathrm{~min} / 0.4 \mathrm{~ms})$ \\
\hline RANS & $167 \mathrm{~s}$ & $31 \mathrm{~ms}$ & $1 \mathrm{~ms}$ & 23.8 years \\
Euler & $92 \mathrm{~s}$ & $31 \mathrm{~ms}$ & $1 \mathrm{~ms}$ & 13.1 years \\
ROM & $0.4 \mathrm{~ms}$ & $31 \mathrm{~ms}$ & $1 \mathrm{~ms}$ & 1.68 days \\
\hline
\end{tabular}

\section{B. Effect of boundary-layer thickness}

The effect of boundary layer thickness is examined for a symmetric case ( $\beta=0 \mathrm{deg})$. Three cases are considered: (1) $\delta / a=0$ (inviscid), (2) $\delta / a=0.08$ and (3) $\delta / a=0.1$. The inviscid results are generated using piston theory.

In the fluid ROM, the structural displacement is represented using the 5 structural modes (the symmetric modes $1,3,5,7,11$ are used). The ranges of parameters are shown in Table 6 . The ranges are all centered at 0.0 , so only the maximum values are given. The ROM is generated using 100 samples.

Figure 12 shows the LCO curves for the different boundary layer thicknesses. The inviscid prediction shows that $\lambda_{c r}=4000.0$, and is not shown in the plot. Decreasing the boundary layer thickness does not have a strong influence on the flutter boundary, $\lambda_{c r}$ decreased by around $1 \%$. However, the boundary layer shows a strong suppression effect on the amplitude of the panel oscillation. At the same $\lambda$, decreasing boundary layer thickness by $20 \%$ would lead to a $100 \%$ increase in the flutter amplitude.

Table 6. Range of parameters for $\operatorname{ROM} \beta=0^{\circ}$

\begin{tabular}{cccccc}
\hline Mode No. & 1 & 3 & 5 & 7 & 11 \\
\hline Range & 0.15 & 0.075 & 0.25 & 0.06 & 0.125 \\
\hline
\end{tabular}




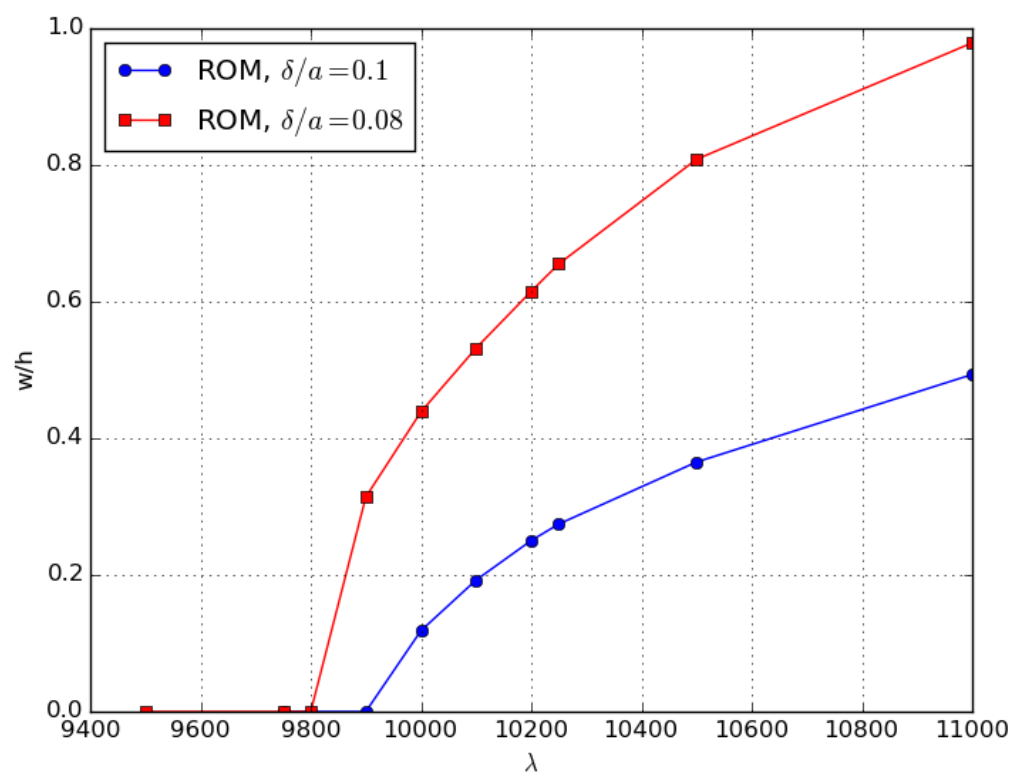

Figure 12. The LCO curves of panel with different boundary layer thicknesses.

\section{Effect of flow orientation angle}

The effect of flow orientation angle is studied for $\delta / a=0$ (inviscid) and $\delta / a=0.1$. Two flow orientation angles are examined $\beta=0,30^{\circ}$.

The ROM for $\beta=30^{\circ}$ requires both symmetric and anti-symmetric modes, so the first 12 structural modes of the panel are used for ROM generation. The ranges of parameters are shown in Table 7. The ranges are all centered at 0.0, so only the maximum values are given. Two sets of data are generated. One data set has smaller ranges of parameter, so that oscillation near undeformed configuration is captured more accurately.

Fig. 13 shows the LCO curves for the different flow orientation angles. Increasing $\beta$ from $0^{\circ}$ to $30^{\circ}$ has a dramatic effect on the flutter boundary. In viscous case, $\lambda_{c r}$ dropped by $40 \%$. In inviscid case, $\lambda_{c r}$ dropped by $70 \%$. The drop of $\lambda_{c r}$ can be explained by the excitation of different modes of the panel at different $\beta$, which are shown in Fig. 14. At $\beta=0^{\circ}$, the dominant modes are the 3rd, 5th, and 7th modes. At $\beta=30^{\circ}$, the 2 nd and 3 rd modes are excited. As modes of lower frequencies are easier to be excited, the panel is more prone to enter flutter.

Table 7. Range of parameters for $\operatorname{ROM} \beta=30^{\circ}$

\begin{tabular}{ccccccccccccc}
\hline Mode No. & 1 & 2 & 3 & 4 & 5 & 6 & 7 & 8 & 9 & 10 & 11 & 12 \\
\hline Data I & 0.6 & 0.5 & 0.4 & 0.3 & 0.2 & 0.2 & 0.2 & 0.2 & 0.2 & 0.2 & 0.2 & 0.2 \\
Data II & 0.1 & 0.15 & 0.1 & 0.03 & 0.08 & 0.08 & 0.03 & 0.015 & 0.015 & 0.015 & 0.035 & 0.015 \\
\hline
\end{tabular}




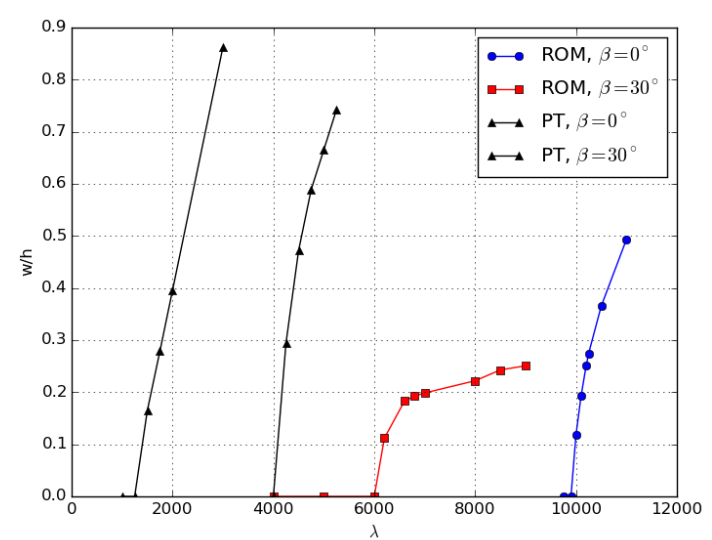

Figure 13. The LCO curves of panel with different flow orientation angles.

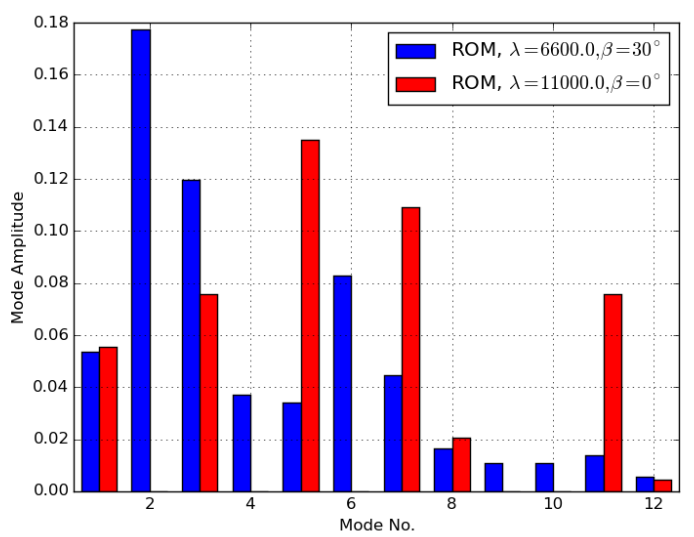

Figure 14. The modal components of responses at different flow orientation angles.

\section{Concluding Remarks}

The computer times required for the aerothermoelastic analysis framework HYPATE were reduced using MOR techniques. The HYPATE framework is extended to include a ROM-based fluid solver, which is based on a PODkriging method. The ROM-based coupled solver is faster than the CFD-based coupled solver by three orders of magnitude. In addition, the smart ordering scheme is shown to reduce the computational cost of the sample generation for ROM by a factor of 2 . The extended framework is verified by comparison with a number of representative test cases.

The framework is applied to hypersonic panel flutter problem by employing the aeroelastic portion of the framework. The study focuses on the effects of boundary layer thickness and flow orientation angle. The boundary layer has minor influence on the flutter boundary, but poses a stabilizing effect on the panel in viscous hypersonic flow. The LCO amplitude of the panel is significantly reduced when the boundary layer thickness is increased. The effect of flow orientation angle is reversed. The flow orientation angle does not have a strong influence on the LCO amplitude, but it can change the critical dynamic pressure dramatically, by exciting different modes of the structure.

\section{Acknowledgement}

The authors would like to acknowledge the help of Professor Jack J. McNamara from The Ohio State University. In addition, the authors are thankful to Intelligent Light ${ }^{\circledR}$ who provided us access to FieldView ${ }^{\circledR}$ post-processing software, which was provided through its University Partners Program.

\section{A. The Algorithm for Smart Ordering Scheme}

The algorithm in the following considers parallel generation of samples, which is necessary when the fluid solver is parallel but without good scalablility. It is more efficient to divide available computational resources into $n_{C}$ groups, and use each group to compute $\frac{1}{n_{C}}$ of the samples at the same time.

1. Use OLH sampling to generate $N_{s}$ samples. An example dataset with two parameters is shown in Fig. 15a. The star indicates the parameter combination for naive flow solution $u_{0}$.

2. Create the k-Nearest-Neighbour (kNN) undirected graph from the sample points, as illustrated in Fig. 15b.

(a) Let the sample points be the vertices of the graph.

(b) For each sample point, find its first $k$ nearest neighbours using the kNN algorithm [23]. The distance in the kNN algorithm is defined using Eq. (40).

(c) Connect the sample points to their nearest neighbours.

(d) If the graph is not fully connected, increase $k$ and go back to step 2.2. Otherwise, continue.

3. Divide the sample points into $n_{C}$ equal-sized groups using standard graph partition algorithm from the METIS package [24], as illustrated in Fig. 15c. 
4. For each group of sample points, represented by a subgraph, generate its minimum spanning tree (MST). The MST of a graph is a subset of its edges that connects all its vertices with the minimum possible total edge length. The MST's are shown in Fig. 15d.

5. Starting from the point that is the closest to zero, determine the dependency of the sample points. The dependency is represented using arrows in Fig. 15d. The sample at the tail of the arrow is the reference solution for the sample at the head of the arrow. In each group, the first sample is restarted using the naive flow solution.

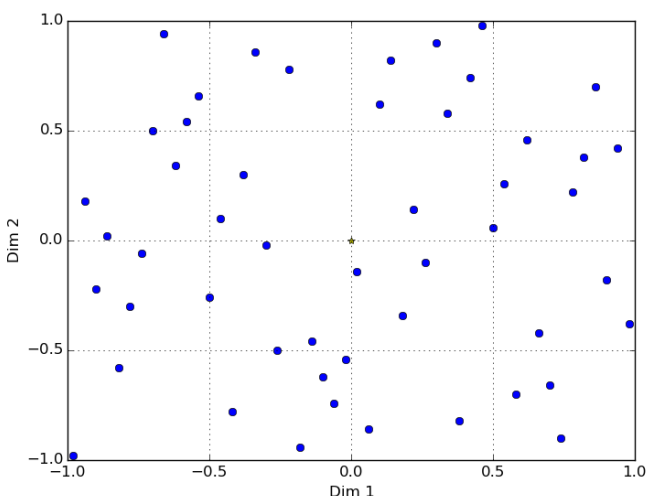

a) The sample data set

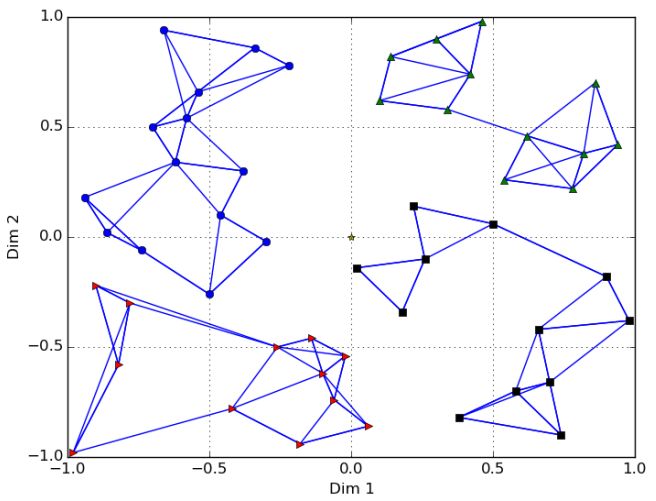

c) The partition of the kNN graph

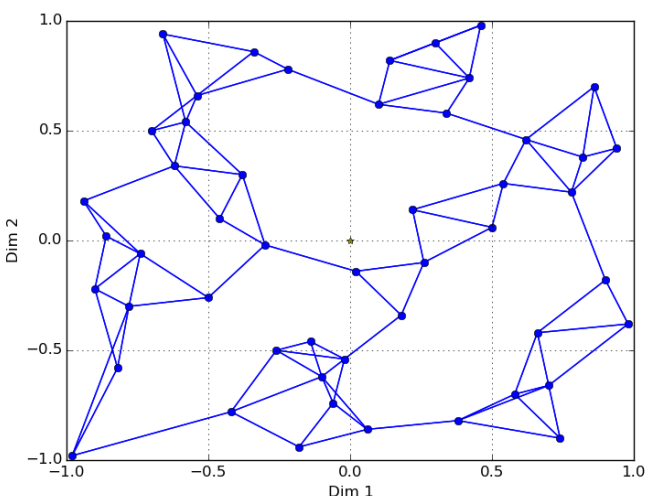

b) The kNN graph of the data set

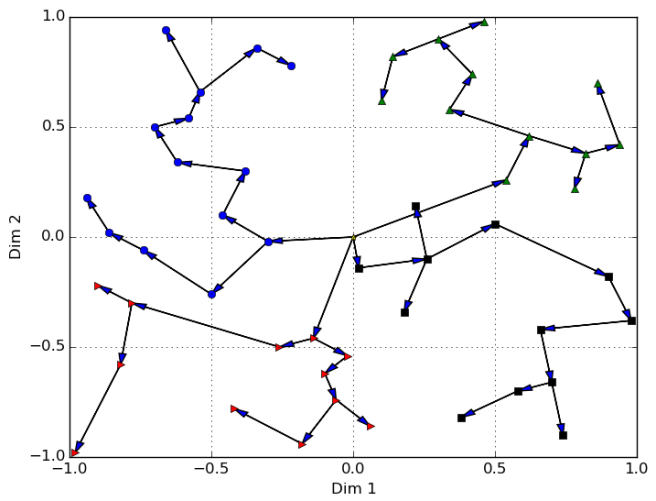

d) The MST's of the subgraphs

Figure 15. Illustration of the smart ordering scheme.

\section{References}

[1] McNamara, J. J. and Friedmann, P. P., "Aeroelastic and Aerothermoelastic Analysis in Hypersonic Flow: Past, Present, and Future," AIAA journal, Vol. 49, No. 6, 2011, pp. 1089-1122.

[2] Crowell, A. R., McNamara, J., and Miller, B., "Hypersonic Aerothermoelastic Response Prediction of Skin Panels Using Computational Fluid Dynamic Surrogates," Journal of Aeroelasticity and Structural Dynamics, Vol. 2, No. $2,2011$.

[3] Falkiewicz, N. J., S. Cesnik, C. E., Crowell, A. R., and McNamara, J. J., "Reduced-Order Aerothermoelastic Framework for Hypersonic Vehicle Control Simulation,” AIAA Journal, Vol. 49, No. 8, 2011, pp. 1625-1646.

[4] Crowell, A. R. and McNamara, J. J., "Model Reduction of Computational Aerothermodynamics for Hypersonic Aerothermoelasticity," AIAA Journal, Vol. 50, No. 1, 2012, pp. 74-84.

[5] Crowell, A. R., McNamara, J. J., Kecskemety, K. M., and Goerig, T., "A Reduced Order Aerothermodynamic Modeling Framework for Hypersonic Aerothermoelasticity," AIAA Paper 2010-2969, 51th AIAA/ASCE/AHS/ASC Structures, Structural Dynamics, and Materials Conference, Jan 2015, pp. 1-22.

[6] Crowell, A. R., Miller, B. A., and McNamara, J. J., "Robust and Efficient Treatment of Temperature Feedback in FluidThermal-Structural Analysis," AIAA Journal, Vol. 52, No. 11, 2014, pp. 2395-2413.

[7] Mei, C., Abdel-Motagaly, K., and Chen, R., "Review of Nonlinear Panel Flutter at Supersonic and Hypersonic Speeds," Applied Mechanics Reviews, Vol. 52, No. 10, 1999, pp. 321-332. 
[8] Friedmann, P. and Hanin, M., "Supersonic Non Linear Flutter of Orthotropic or Isotropic Panels with "arbitrary" Flow Direction," Israel Journal of Technology, Vol. 6, 1968, pp. 46-57.

[9] Huang, D. and Friedmann, P. P., "An Integrated Aerothermoelastic Analysis Framework for Predicting the Response of Composite Panels," AIAA 2016-1090, 15th Dynamics Specialists Conference, San Diego, CA, Jan. 2016, pp. 1-37.

[10] Balay, S., Abhyankar, S., Adams, M., Brown, J., Brune, P., Buschelman, K., Eijkhout, V., Gropp, W., Kaushik, D., Knepley, M., et al., "PETSc Users Manual Revision 3.7," Tech. rep., Argonne National Laboratory, 2016.

[11] Jameson, A., "Solution of the Euler Equations for Two Dimensional Transonic Flow by a Multigrid Method," Applied Mathematics and Computation, Vol. 13, No. 3-4, 1983, pp. 327-355.

[12] Culler, A. J. and McNamara, J. J., "Impact of Fluid-Thermal-Structural Coupling on Response Prediction of Hypersonic Skin Panels," AIAA Journal, Vol. 49, No. 11, 2011, pp. 2393-2406.

[13] Forrester, A., Sobester, A., and Keane, A., Engineering Design Via Surrogate Modelling: A Practical Guide, John Wiley \& Sons, 2008.

[14] Simpson, T. W., Poplinski, J., Koch, P. N., and Allen, J. K., "Metamodels for Computer-Based Engineering Design: Survey and Recommendations," Engineering With Computers, Vol. 17, No. 2, 2001, pp. 129-150.

[15] Bryan Glaz, Active/Passive Optimization of Helicopter Rotor Blades for Improved Vibration, Noise, and Performance Characteristics, Ph.D. thesis, University of Michigan, Ann Arbor, 2008.

[16] Sirovich, L., "Turbulence and the Dynamics of Coherent Structures, Part I: Coherent Structures," Quarterly of Applied Mathematics, Vol. 45, No. 3, 1987, pp. 561-571.

[17] Lucia, D. J., Beran, P. S., and Silva, W. A., "Reduced-Order Modeling: New Approaches for Computational Physics," Progress in Aerospace Sciences, Vol. 40, No. 1, 2004, pp. 51-117.

[18] Ashley, H. and Zartarian, G., "Piston Theory - a New Aerodynamic Tool for The Aeroelastician," Journal of the Aeronautical Sciences, Vol. 23, No. 12, 1956, pp. 1109-1118.

[19] Dowell, E. H., Aeroelasticity of Plates and Shells, Vol. 1, Springer Science \& Business Media, 1974.

[20] Gordnier, R. E. and Visbal, M. R., "Development of a Three-Dimensional Viscous Aeroelastic Solver for Nonlinear Panel Flutter," Journal of Fluids and Structures, Vol. 16, No. 4, 2002, pp. 497-527.

[21] Lock, M. H., "A Note on the Application of the Quasi-Steady Supersonic-Flow Theory to Flutter Analysis," Journal of the Aerospace Sciences, 2012.

[22] Hashimoto, A., Aoyama, T., and Nakamura, Y., "Effects of Turbulent Boundary Layer on Panel Flutter," AIAA Journal, Vol. 47, No. 12, 2009, pp. 2785-2791.

[23] Altman, N. S., "An Introduction to Kernel and Nearest-Neighbor Nonparametric Regression," The American Statistician, Vol. 46, No. 3, 1992, pp. 175-185.

[24] Karypis, G. and Kumar, V., "A Fast and High Quality Multilevel Scheme for Partitioning Irregular Graphs," SIAM Journal on Scientific Computing, Vol. 20, No. 1, 1998, pp. 359-392. 